\title{
Reflets
}

Revue ontaroise d'intervention sociale et communautaire

\section{Spiritualité, guérison et autonomie gouvernementale dans le contexte politique Ojibwas}

\section{François Boudreau et Herbert Nabigon}

Volume 6, numéro 1, printemps 2000

Approches d'intervention : définir et renouveler nos pratiques

URI : https://id.erudit.org/iderudit/026297ar

DOI : https://doi.org/10.7202/026297ar

Aller au sommaire du numéro

Éditeur(s)

Reflets : Revue ontaroise d'intervention sociale et communautaire

ISSN

1203-4576 (imprimé)

1712-8498 (numérique)

Découvrir la revue

Citer cet article

Boudreau, F. \& Nabigon, H. (2000). Spiritualité, guérison et autonomie gouvernementale dans le contexte politique Ojibwas. Reflets, 6(1), 108-127.

https://doi.org/10.7202/026297ar

Tous droits réservés (C) Reflets : Revue ontaroise d'intervention sociale et communautaire, 2000
Ce document est protégé par la loi sur le droit d'auteur. L'utilisation des services d'Érudit (y compris la reproduction) est assujettie à sa politique d'utilisation que vous pouvez consulter en ligne.

https://apropos.erudit.org/fr/usagers/politique-dutilisation/ 


\section{Spiritualité, guérison et autonomie gouvernementale dans le contexte politique Ojibwas}

François Boudreau,

École de service social, Université Laurentienne et

Herbert Nabigon,

Nishnaabe Kinoomaadwin Naadmaadwin, Université Laurentienne

\section{Répression, renaissance culturelle et enjeux politiques}

Aujourd'hui, lorsque l'on parle des populations amérindiennes, il est de mise de parler d'une renaissance politique et culturelle ${ }^{1}$ se situant à plusieurs niveaux (Schmalz 1991). Cette renaissance consiste en une reprise en main des Premières Nations par ellesmêmes, en une réappropriation de leurs cultures et, à un niveau tout aussi fondamental, en un renouvellement complet des pratiques d'intervention au sein des communautés. D'abord, l'intervention est maintenant le fait d'autochtones qui connaissent leur culture. Il y a de moins en moins d'interventions extérieures, de gens qui pensent connaître, mais qui brouillent en fait les enjeux de l'intervention. Ensuite, l'intervention ne se résume pas à la dimension du service social, loin de là, elle est le prolongement d'une reprise en main politique, l'expression d'une volonté d'autodétermination et d'un renouveau de la culture, le tout informé d'une spiritualité à caractère ancestral. Les pratiques 
d'intervention ont aujourd'hui un caractère résolument Anishnabee ${ }^{2}$. Cette renaissance culturelle est également politique et cela a des implications importantes pour les populations euroaméricaines $^{3}$ (CRPA 1996a).

Concrètement, ce processus devrait aller jusqu'à un réaménagement des structures politiques du Canada. Mais cela signifie aussi que toutes les discussions, tant au niveau des politiques sociales qu'à celui de l'intervention auprès des Premières Nations, doivent reconsidérer l'histoire des relations entre les peuples venus d'ailleurs et les peuples d'ici. La culture dominante euro-américaine doit remettre en question les conceptions méprisantes, assimilatrices et paternalistes qu'elle a trop longtemps maintenues à l'égard des Amérindiens.

Le Canada n'a pas d'héritage colonial hors de ses frontières, mais il en a à l'intérieur. La culture euro-américaine du Canada doit revoir tous ses rapports, tous les arrangements et toutes les ententes politiques, nommément les traités et les conditions de vie des populations autochtones. Elle doit aussi revoir son histoire dans le but de remédier aux effets que cet héritage colonial interne a fait subir aux Anishnabee. Ces discussions devraient favoriser une stratégie qui permettrait aux Premières Nations de regagner leur place légitime dans la structure politique du Canada. Cette place est celle des "gardiens de cette terre» (Ghostkeeper 1995 : 42), elle-même désignée du nom de l'île de la Tortue. La reconnaissance politique faciliterait le processus de guérison

"Les aînés informent les Anishnabee que le temps de cette guérison est arrivé, que le temps est venu de relever la tête pour approfondir le mouvement de renaissance amorcé et pour assurer l'accomplissement de l'autodétermination". entrepris au sein de plusieurs Premières Nations. Les aînés informent les Anishnabee que le temps de cette guérison est arrivé, que le temps est venu de relever la tête pour approfondir le mouvement de renaissance amorcé et pour assurer l'accomplissement de l'autodétermination. Cela signifie donc que le temps est également venu pour la société euro-américaine d'écouter ce que disent les Anishnabee et faire l'effort de comprendre ce qu'ils ont à nous dire.

Dans ce texte, nous présentons certaines idées sur l'autonomie gouvernementale et nous indiquons comment l'application des enseignements traditionnels fondés sur les couleurs sacrées de la roue médicinale peuvent aider aux Anishnabee à regagner leur 
fierté et leur dignité parmi les peuples de la terre. La réintroduction de ce savoir traditionnel compte parmi les plus importantes initiatives éducationnelles et méthodes d'intervention sociale, caractéristiques de cette renaissance culturelle Anishnabee. Nous tenterons, par la structure de l'exposé, de faire comprendre au lecteur comment l'autodétermination, la renaissance culturelle et le renouvellement des pratiques d'intervention sont le prolongement d'une spiritualité globale, au sens de non-séparation entre ce que la culture euro-américaine a distingué comme le pouvoir temporel (société politique) et le pouvoir religieux (l'Église).

\section{Sur une histoire des relations}

Du point de vue Anishnabee, la Proclamation Royale de 1763 demeure la Magna Carta des relations entre les peuples amérindiens et les Euro-Canadiens dans ce pays connu sous le nom de Canada. Au temps de la Proclamation Royale, les peuples amérindiens détenaient la balance du pouvoir économique, social, politique et militaire dans toute la région centrale de l'Amérique du Nord. Le contenu de la Proclamation Royale indique que les Premières Nations, le gouvernement colonial et les colons consentaient à coexister en paix. La coexistence pacifique ainsi envisagée a existé, tant bien que mal, jusqu'à la création de la province du Manitoba en 1870. Mais déjà, après la guerre canado-américaine de 18121814, l'État du Canada commençait à sentir que le rapport de force tournait en sa faveur. Le temps où les Amérindiens était des alliés indispensables tirait à sa fin et cela, d'une part, parce que la paix continentale semblait assuré et, d'autre part, parce que l'augmentation du nombre de colons diminuait dramatiquement le poids démographique relatif des Amérindiens. D'environ un demi-million d'habitants en 1814, la population euro-américaine est passé à 4 millions environ en 1870. L'inverse s'est produit avec les Anishnabee. D'environ 2,2 millions d'individus au moment du contact, leur nombre est passé à 102358 en 1871. Quant au nombre 
"...l'État canadien

n'a jamais reconnu la

légitimité des

revendications

politiques métisses et amérindiennes».

"Ces politiques

ségrégationnistes, d' "apartheid", ont laissé leurs marques, encore visibles aujourd'hui...» de métis, il est estimé à 30000 vers 1871. Ce n'est qu'en 1991 que le nombre combiné des populations Anishnabee et Métis a atteint à nouveau le million (Denevan 1976; Ubelaker 1976).

La création de la province du Manitoba, qui fait suite aux conflits entre l'État et le chef métis Louis Riel (1844-1885) et son allié le chef Cris Poundmaker (1842-1886), aussi connu sous le nom de Pitikwahanapiwiyin, a marqué la rupture de la coexistence pacifique entre les Euro-Américains et les Amérindiens. Riel et Poundmaker étaient engagés dans la lutte pour l'établissement politique et territorial des Métis et des Amérindiens à l'ouest des deux Canada ${ }^{4}$. Ces deux hommes ont été des Pères de la Confédération, au même titre que John A. McDonald (Haut Canada) et George Étienne Cartier (Bas Canada). Cependant, l'État canadien n'a jamais reconnu la légitimité des revendications politiques métisses et amérindiennes. Les livres n'ont retenu, de leur contribution à l'histoire du Canada, que les accusations de trahisons portées contre ces deux hommes. Riel a été pendu en 1885, tandis que Poundmaker a été incarcéré à la prison de Stoney Mountain et il est mort quatre mois après sa libération, en 1886. Suite à la défaite des Métis de Riel et des Amérindiens de Poundmaker, la balance du pouvoir s'est complètement déplacée vers Ottawa et le poids politique amérindien a été totalement anéanti.

Le gouvernement du Canada a alors entrepris 125 années de politiques d'assimilation et d'isolement, qui ont résulté en une très forte acculturation et, dans certaines communautés amérindiennes, à une déstructuration complète de la culture. Ces politiques ségrégationnistes, d'«apartheid», ont laissé leurs marques, encore visibles aujourd'hui, dans plusieurs communautés des Premières Nations. Le suicide, les mutilations, la violence, la toxicomanie et le décrochage scolaire comptent parmi les principaux problèmes (CRPA 1996b; CRPA 1996c; 1996d; CRPA 1995; Shkilnyk 1985). 


\section{Les Premières Nations en quelques chiffres}

Lorsque l'on considère les conditions des Premières Nations à partir des chiffres, la situation ressemble à celle des pays en développement. Par exemple, $65 \%$ des terres aborigènes au Canada se trouvent dans des régions éloignées, inaccessibles par route et ayant très peu de valeur commerciale (Ross 1987). Il sera très difficile pour les peuples amérindiens d'acquérir une indépendance économique s'il n'y a rien sur leurs terres à développer, si les terres «réservées» sont trop petites ou si elles sont éloignées des réseaux de communications (CRPA 1996b). Par conséquent, plus de territoires mieux connectés aux réseaux routiers sont nécessaires au développement économique et à l'émancipation politique des Anishnabee. Les revendications territoriales sont donc un moyen essentiel à l'acquisition d'une base territoriale viable, nécessaire à leur autodétermination.

On dénombre aujourd'hui plus de 600 communautés amérindiennes au Canada. Elles sont dispersées en autant de réserves. Chacune de ces communautés a besoin de construire dix nouvelles maisons et d'en rénover dix autres, pour un besoin total de 12,000 unités de logement (Knox 1990), afin de répondre aux besoins pressants de la situation. Même si le Canada a dépensé deux milliards de dollars dans les dix dernières années pour le logement amérindien, la situation demeure pitoyable (CRPA 1996c). À vrai dire, le gouvernement du Canada ne semble pas avoir de stratégie, ni de politique pour résorber cette crise du logement. Les conséquences sont graves. Par exemple,

Même si le taux de mortalité infantile diminue parmi les enfants aborigènes, il demeure bien au-dessus de la moyenne nationale. La mortalité infantile est due aux affections respiratoires et aux maladies infectieuses et parasitaires, ce qui reflète des logements de pauvre qualité, le manque de système d'égouts et d'eau potable, ainsi qu'un accès limité aux institutions médicales (Ross 1987 :44). 
"Les taux de chômage des communautés des Premières Nations varient entre $50 \%$ et $90 \%$, selon les communautés...»
Le chômage demeure également un grave problème. Les taux de chômage des communautés des Premières Nations varient entre $50 \%$ et $90 \%$, selon les communautés (CRPA 1996e). Ce qui est encore plus sérieux, c'est que les peuples aborigènes sont les derniers embauchés pendant les périodes de croissance économique et les premiers licenciés lors des périodes de crise (Angus 1991). Même pendant les périodes de croissance, des employeurs situés près des réserves préfèrent embaucher des travailleurs d'autres régions. Cela est notamment vrai dans le Nord de l'Ontario, comme l'ont montré les démêlés de la Première Nation de Constance Lake, près de Hearst, avec la compagnie Trans-Canada Pipeline. Cette dernière voulait construire un pipeline sur les terres amérindiennes sans se rendre aux revendications des Anishnabee de Constance Lake. Ces derniers exigeaient en retour qu'un certain nombre d'emplois qualifiés et rémunérateurs soit réservé aux membres de cette Première Nation. Les emplois disponibles aux Amérindiens sont généralement des emplois saisonniers, même dans les meilleurs moments du cycle économique (CRPA 1996e). Pour nous, cela demeure une des formes insidieuses de racisme institutionnel. L'indice statistique le plus inquiétant concerne cependant le nombre de suicide chez les jeunes.

Au Canada, il y a environ 19 suicides par cent mille $(100,000)$ personnes situées dans la tranche d'âge de quinze (15) à vingt-quatre (24) ans. Dans cette même tranche, il $y$ a cent trente (130) suicides par cent mille (100 000) personnes parmi la population aborigène (Ross 1987 : 45).

Ce qui est alarmant, c'est que $38 \%$ de la population amérindienne a moins de 15 ans. Si la tendance au suicide se maintient, c'est à une hécatombe que nous devrions assister dans la prochaine décennie (CRPA 1995 : 12-20).

Ce portrait inquiétant force à conclure que la pauvre qualité des conditions de vie a un impact physique et émotif très profond chez les Anishnabee. Les gens expriment souvent leur douleur et leur désarroi en ayant recours, entre autres, à la violence. Toutes 
les pathologies sociales qui affectent les Anishnabee sont bien documentées dans les nombreuses publications et les rapports de recherches. Des solutions ont été proposées par les Premières Nations et des sympathisants à la cause amérindienne (CRPA 1996d). Mais l'État fait encore trop peu et trop lentement. Les différents paliers de gouvernement au Canada doivent faire davantage sur le front politique. Ils pourraient imiter l'exemple du gouvernement de l'Ontario qui, le 6 août 1991, a décidé de reconnaitre les Premières Nations comme des «nations domestiques» ${ }^{5}$. Ceci a été et continue d'être une décision importante et symbolique prise par le gouvernement de l'Ontario de l'époque, même si l'actuel gouvernement conservateur a réintroduit l'attitude raciste et méprisante d'antan.

\section{Une stratégie pour mettre en œuvre l'autonomie gouvernementale}

Afin d'assurer le respect des institutions sociales et économiques des Premières Nations, le gouvernement fédéral devrait considérer le cadre suivant comme un moyen pour établir une relation fonctionnelle et formelle avec les Premières Nations.

- Il devrait reconnaitre la nécessité d'un «testament national» identifiant la division des pouvoirs. Ces pouvoirs seraient négociés entre le gouvernement fédéral et les Premières Nations. Un processus bilatéral spécial devrait être immédiatement établi pour conclure un accord.

- Un processus continu d'amendements et de révisions constitutionnels devrait être mis en place, en assurant une participation permanente des Premières Nations.

- Les revendications territoriales non résolues devraient faire l'objet d'une attention spéciale et des mécanismes permanents d'application des accords sur les revendications territoriales devraient être prévus. 
- Le développement économique devrait être prioritaire et les investissements intensifiés pour réduire la pauvreté.

- Les services sociaux essentiels de qualité raisonnable devraient être disponibles pour tous les Amérindiens, sans considération de statut.

- Les Premières Nations devraient être capables de générer des recettes fiscales qui amélioreraient les services aux individus et qui favoriseraient leur autonomie. À long terme, ces prélèvements fiscaux réduiraient leur dépendance à l'égard des fonds fédéraux.

- Les droits individuels devraient être protégés et respectés dans le contexte du respect des droits collectifs des Premières Nations.

- Les Amérindiens devraient jouir, sans considération de statut, de chances égales dans tous les domaines de la vie collective.

Toutes ces recommandations sont déjà contenues dans le rapport de la Commission Royale sur les Peuples Autochtones (CRPA 1996e : 161-289), mais le gouvernement fédéral a décidé de ne

"La solution à la résolution des problèmes et à la réalisation des aspirations politiques des Premières Nations manque définitivement de volonté $[\ldots]$ politique de la part $d u$ gouvernement central». pas y donner suite et de rayer le rapport de ses tablettes. La solution à la résolution des problèmes et à la réalisation des aspirations politiques des Premières Nations manque définitivement de volonté politique de la part du gouvernement central.

À titre indicatif, mentionnons que les États-Unis considèrent les communautés amérindiennes vivant sur son territoire comme souveraines dans leurs affaires internes. Les Premières Nations et le Canada pourraient considérer l'expérience américaine et adopter un point de vue semblable à celui présenté par Kickingbird et al. :

La loi est claire, toutefois, qu'une nation amérindienne possède tous les pouvoirs inhérents à tout gouvernement souverain, exception faite des cas où ces pouvoirs ont été qualifiés ou limités par des traités, des accords ou des lois spécifiques $d u$ Congrès. Par conséquent, si des tribus ont perdu certains de leurs pouvoirs, elles maintiennent tous les autres. Donc, elles peuvent et exercent plusieurs pouvoirs souverains. $(1977: 8)$ 
Les pouvoirs revendiqués par les Premières Nations sont les pouvoirs inhérents à toute forme d'autodétermination. Ces pouvoirs sont ancestraux et, contrairement à une interprétation à la mode dans les cercles politiques, les Premières Nations n'ont jamais abdiqué ces pouvoirs. Les pouvoirs inhérents à tout gouvernement amérindien sont les suivants.

- Le pouvoir de déterminer la forme du gouvernement.

- Le pouvoir de définir les conditions d'adhésion à la nation.

- Le pouvoir d'administrer la justice et de faire respecter les lois.

- Le pouvoir de taxation.

- Le pouvoir de régler les relations domestiques de ses membres.

- Le pouvoir de régler l'utilisation de la propriété.

Ces pouvoirs se concrétisent par des droits.

- Le droit de passer des lois, d'interpréter les lois et d'administrer la justice.

- Le droit de définir les pouvoirs et les responsabilités des fonctionnaires.

- Le droit de déterminer si les lois conçues au nom du gouvernement amérindien par le gouvernement fédéral font autorité.

- Le droit de définir la manière dont les fonctionnaires gouvernementaux seront sélectionnés et destitués.

\section{Un fondement à l'autodétermination}

Jamais les Premières Nations n'ont abdiqué leurs droits à l'autodétermination. Les droits aborigènes au Canada reposent sur trois fondements juridiques : la Proclamation Royale de 1763, le droit international et le droit communal. Mais la société dominante, depuis la fin de la Guerre de 1812-1814, a toujours tenté de diminuer et de restreindre les droits autochtones. La Proclamation Royale de 1763 a été restreinte à une «expression historique du droit communal, plutôt que [d'être considérée] comme source de droits légitimes» (Platiel 1992 : 6). Bien que nettement renforcée par la décision 
"Depuis longtemps et de manière éloquente, les dirigeants politiques des Premières Nations ont justifié leur besoin à l'autodétermination, mais les gouvernements euroaméricains n'ont pas encore daigné reconnaître ce besoin».
Sparrow $^{6}$, la définition juridique des droits aborigènes demeure faible et fragile. Les tribunaux exigent la preuve que l'occupation et l'utilisation territoriale spécifique - chasse, pêche, rites cérémoniels, etc. - précède la colonisation européenne. On voit le problème que cela pose à des peuples de tradition orale jadis nomades dont presque tous les artefacts étaient en os, en bois ou en peau et qui se sont décomposés depuis longtemps. Mais nous pensons qu'il n'appartient pas aux tribunaux d'interpréter la loi pour savoir si, oui ou non, les Premières Nations ont le droit à l'autonomie gouvernementale et à l'usage territorial. La meilleure stratégie pour l'obtention de la reconnaissance à l'autodétermination est de mener une négociation politique. Les Premières Nations doivent exiger la possession complète et souveraine de leurs terres, des ressources, des droits culturels et la reconnaissance légale du droit coutumier et du droit inhérent à l'autonomie gouvernementale.

Depuis longtemps et de manière éloquente, les dirigeants politiques des Premières Nations ont justifié leur besoin à l'autodétermination, mais les gouvernements euro-américains n'ont pas encore daigné reconnaître ce besoin. Les aînés reprennent ce point avec insistance et expliquent que le fondement à l'autodétermination est spirituel. Cela prête en général à confusion au sein des populations euro-américaines, voire même chez plusieurs Amérindiens. Mais les droits aborigènes ne proviennent pas des lois, des institutions ou des idées établies par les gouvernements, ils sont un don de Kitchi Manido, le Grand Esprit Créateur de l'île de la Tortue. Jusqu'à ce que ce soit compris et accepté par les Euro-Américains, la reconnaissance des droits aborigènes demeurera problématique. La terre et le mode de vie qui l'accompagne sont aussi des dons de Kitchi Manido et personne ne peut les enlever. Ces dons ne peuvent pas être ignorés, rationalisés ou oubliés, ils demeurent éternels, «aussi longtemps que brillera le soleil et que couleront les rivières».

Parmi les dons du Créateur, on trouve les quatre directions sacrées. Elles sont utilisées dans la quête de l'harmonie et de la paix intérieure. Mises en application, ces quatre directions sacrées fournissent des conseils aux leaders spirituels des Premières Nations 
dans leur tentative de recréer les formes traditionnelles de gouvernement.

Le rôle des ainés Anishnabee est d'offrir des conseils spirituels, tandis que le rôle des dirigeants politiques est de voir au bienêtre du peuple. Les dirigeants politiques tentent de traduire les valeurs et les enseignements traditionnels aux représentants gouvernementaux euro-américains. Beaucoup se rappellent les paroles d'Ovide Mercredi lorsqu'il a été élu grand chef de l'Assemblée des Premières Nations, à l'été 1991. Dans son discours d'acceptation, il disait qu'il écouterait attentivement les aînés et qu'il serait guidé par la perspicacité de leurs idées et de leurs conseils. Les aînés sont considérés comme étant le fondement du leadership. Ces mêmes paroles ont été reprises par Philippe Fontaine, en avril 1997, lorsqu'il a succédé à Ovide Mercredi.

\section{Le paradigme culturel}

"[...] les communautés autochtones ont développé une série de réponses culturelles, allant de la conservation des valeurs traditionnelles jusqu'à l'adoption de valeurs résolument modernes, en passant à mi-chemin par une adaptation mixte entre la tradition et la modernité».
La cohérence de la vie culturelle, sociale, économique, politique et géographique ${ }^{7}$ a été très durement éprouvée par les politiques souvent hostiles de l'État canadien. On a longtemps parlé de "civiliser les sauvages» en les assimilant aux valeurs de la culture dominante. En réponse à ces conditions, les communautés autochtones ont développé une série de réponses culturelles, allant de la conservation des valeurs traditionnelles jusqu'à l'adoption de valeurs résolument modernes, en passant à mi-chemin par une adaptation mixte entre la tradition et la modernité. Les croyances, les pratiques culturelles et les traditions sont demeurées relativement intactes dans plusieurs milieux traditionnels. Toutefois, dans les communautés adaptées à la vie contemporaine, plusieurs ont perdu les valeurs de leur culture. C'est pourquoi un processus de revitalisation culturelle prenant appui sur les enseignements des aînés a été introduit, ce que plusieurs nomment la renaissance. La réappropriation de la culture ou de ce qui en reste découle directement de cette revalorisation. Tout cela a largement été le résultat du travail des aînés. Plusieurs éléments de la culture ont 
"C'est dans la réappropriation de la culture ancestrale que la jonction des niveaux politique et spirituel des activités autochtones s'effectue..." donc été réappropriés, mis en pratique et intégré au quotidien comme la langue (CRPA 1996b), le jeûne, le don, la cérémonie du sauna (sweat lodge), l'usage de la roue médicinale, du «paradigme culturel» (Nabigon 1992 : 140-144) et des plantes médicinales, la fumigation avec la sauge, l'usage du sentencing circle (Ross 1996; Boudreau et Ribordy 1999), etc., comptent parmi ces éléments de culture appropriés de nouveau. C'est dans la réappropriation de la culture ancestrale que la jonction des niveaux politique et spirituel des activités autochtones s'effectue (Boudreau 2000). Il faudra même comprendre que c'est la spiritualité des aînés qui informe le contenu politique des activités.

Il importe donc à la société euro-américaine de comprendre les valeurs, les attitudes et la vision du monde qui caractérisent en général les autochtones. La philosophie sur laquelle se fonde le paradigme culturel reconnait et reflète une vision traditionnelle du monde. Cette vision interprète les relations communautaires conformément aux préceptes culturels autochtones. L'interprétation spirituelle de cette vision est divisée en quatre directions sacrées (Figure 1). C'est cette vision traditionnelle ou ancestrale de la culture que nous vous présentons maintenant ${ }^{8}$. Cette vision, cette manière d'appréhender le monde est aujourd'hui utilisée pour aborder les problèmes des individus et des communautés. Mais surtout, cette vision du monde est utilisée dans la guérison et dans la réinsertion sociale des Anishnabee.

\section{L'Est}

«Le Créateur a engendré la vie au printemps, à l'Est qui est symbolisé par la conleur ronge».
Le Créateur a engendré la vie au printemps, à l'Est, qui est symbolisé par la couleur rouge. Les peuples autochtones croient qu'ils ont été représentés par l'Est qui marque également le début. Le Créateur a accordé aux peuples le don de la nourriture, des sentiments et de la vision. Le vent d'Est souffle la vie nouvelle sur notre Mère la Terre. Le rouge est le symbole du renouvellement. Le printemps nous apprend à être reconnaissants envers la vie.Au printemps, les animaux donnent naissance aux petits. Ces animaux sont à la fois nos 
enseignants et notre nourriture. Il existe une forte relation entre la nourriture et les sentiments. Une bonne nourriture contribue à de bons sentiments. Lorsque nous nous sentons bien, nous profitons de la vision. À l'opposé des bons sentiments, se trouvent les sentiments d'infériorité. Aujourd'hui, beaucoup d'Amérindiens se sentent inférieurs et une colère intense monte de ce sentiment d'infériorité. La colère qui vient de l'Est serait à l'origine de plusieurs des problèmes familiaux déjà évoqués. L'enseignement traditionnel provenant de cette direction porte un message de paix et d'harmonie pour les communautés. Ne pas tenir compte de cette vérité dans l'effort pour recréer un sens d'autodétermination, serait comme essayer de construire une maison sans fondation solide.

\section{Figure 1 : Interprétation spirituelle des directions sacrées}

NORD

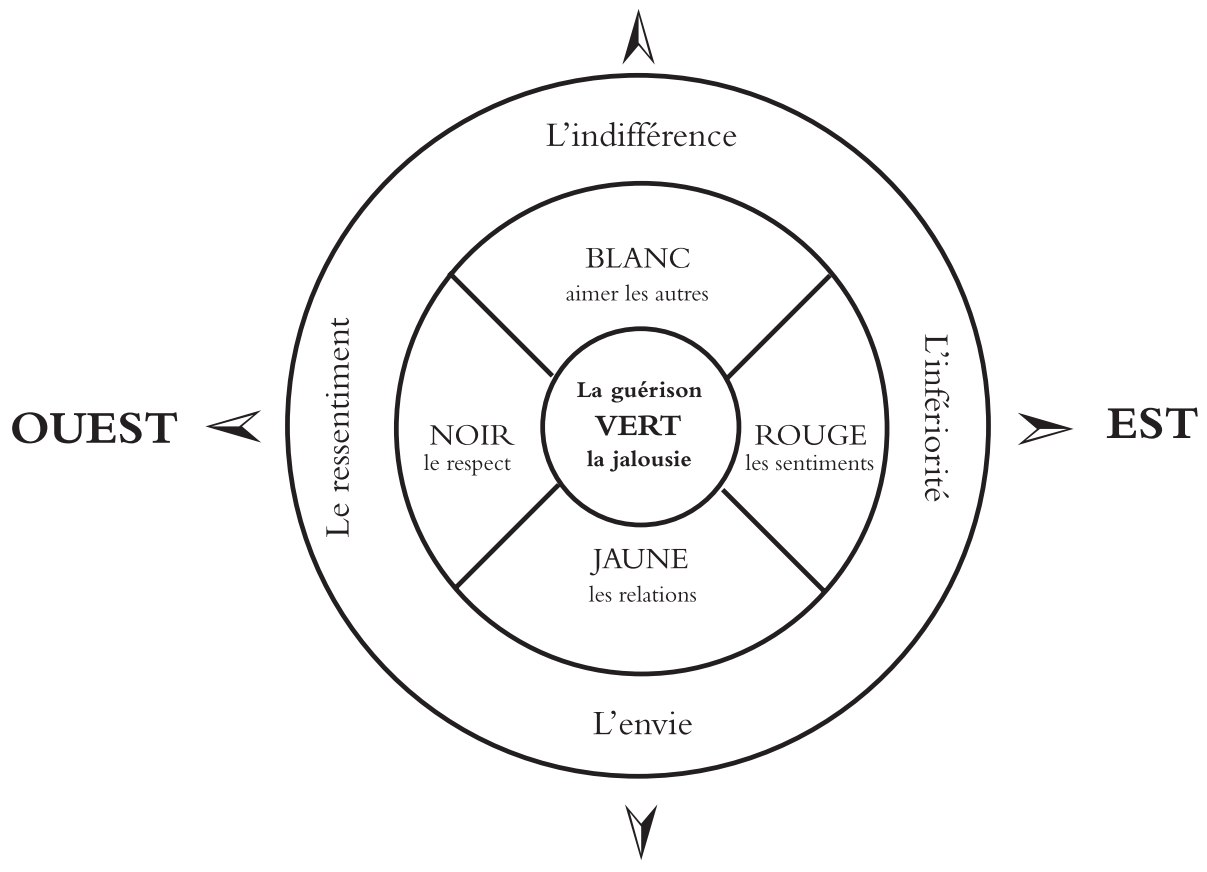

SUD 


\section{Le Sud}

"La couleur jaune symbolise l'été, le temps, les relations et le soleil».
La couleur jaune symbolise l'été, le temps, les relations et le soleil. À midi, le soleil est au sud. La chaleur de l'été nous apprend à être patient.Le développement de soi est considéré comme étant essentiel aux jeunes. Le jaune aide à faire la lumière en soi-même, à se comprendre, à l'aide de la famille, de la famille élargie, des amis et de la communauté. Cela prend du temps pour comprendre notre identité d'être humain. Nous apprenons et comprenons notre «être» dans l'interaction avec nos pairs et par l'acquisition des valeurs qui nous sont transmises par nos parents. En ces temps modernes, les adolescents amérindiens peuvent éprouver une période extrêmement difficile à cause du contexte biculturel dans lequel ils vivent. La puberté est une période de changement pour tous les adolescents. L'adolescence est souvent une période de crise. Pour les jeunes amérindiens, c'est alors le temps de redéfinir leur amérindianité. Le processus d'acquisition de leur héritage culturel a alors préséance sur toutes autres activités, y compris l'éducation institutionnelle. Il n'est donc pas étonnant que leurs résultats scolaires tendent à chuter lorsqu'ils sont immergés dans les systèmes d'éducation euro-américains qui imposent ses valeurs et sa vision du monde. Souvent les enseignants et les parents oublient de prendre ces facteurs en considération lors d'une crise scolaire. Les aînés et les enseignants traditionnels aident les adolescents à comprendre et à désamorcer la crise. Ces adolescents sont notre prochaine génération de dirigeants et un retour aux manières et aux visions traditionnelles, supportées et encouragées par la communauté, est un élément essentiel dans la fondation spirituelle de l'autodétermination.

\section{L'Ouest}

«La couleur noire représente l'automne, le respect, la raison et l'eau».
La couleur noire représente l'automne, le respect, la raison et l'eau. Nos corps sont largement composés d'eau et, par conséquent, cela nous unit aux rivières et aux lacs. L'eau nous aide à voir plus 
clairement. Le respect signifie de penser à deux fois avant de poser le geste. La réalité de la vie intérieure s'améliore lorsque l'on comprend et que l'on met en pratique le «respect». Beaucoup d'adolescents ont des problèmes à revoir leur vie intérieure à cause des changements et des crises qu'ils traversent. Les chefs spirituels amérindiens ont une compréhension intime de l'adolescence et des cérémonies de guérison sont disponibles pour aider les adolescents. Parfois les familles dysfonctionnelles accablent les enfants aînés de la responsabilité de prendre soin des cadets et des cadettes. Cette pratique crée de l'hostilité et du ressentiment chez ces jeunes. Les sentiments d'amertume détruisent la dignité personnelle. En fin de compte, comment pouvons-nous, en tant que Premières Nations, nous gouverner comme Nations lorsque nous luttons pour regagner le contrôle de nos propres familles? Nous avons besoin de regagner notre équilibre pour que nos communautés puissent aller de l'avant, et cela doit être appuyé sur le respect.

\section{Le Nord}

"La couleur blanche représente l'hiver, le mouvement, l'air, le souci des autres et la paix. En hiver, la terre se repose et les animaux dorment».
La couleur blanche représente l'hiver, le mouvement, l'air, le souci des autres et la paix. En hiver, la terre se repose et les animaux dorment. Le souci des autres peut être mesuré par le niveau d'interactions dans le cadre de la famille, de l'école, de la communauté et de la nation. L'isolement indique ordinairement que des problèmes existent et, conséquemment, que l'on doit s'en occuper. Il faut normalement développer un plan d'action dans lequel on expose le comportement dans le but de briser l'isolement et l'absence de désir qui l'accompagne et de progressivement amener la personne à prendre soin d'elle-même et à assumer ses responsabilités. Les aînés et les enseignants traditionnels comprennent la nature sacrée de l'amour pour l'autre et ses implications. Il faut comprendre la manière dont nous interagissons et comment nous nous comportons les uns envers les autres et, au besoin, nous devons mobiliser les niveaux 
appropriés d'intervention. Une fois encore, sans cet élément important du souci de l'autre, le fondement de l'autodétermination demeure faible.

\section{Au Centre}

"Le vert est une couleur curative qui représente notre Mère la Terre. Le vert représente également l'équilibre, l'harmonie et l'écoute».
Le vert est une couleur curative qui représente notre Mère la Terre. Le vert représente également l'équilibre, l'harmonie et l'écoute. La Terre nourrit toutes les couleurs et tous les êtres vivants. Les chefs spirituels insistent pour que nous écoutions et prêtions attention aux côtés sombres de la vie. Les côtés sombres peuvent se définir par cinq pièges : l'infériorité, l'envie, le ressentiment, l'indifférence et la jalousie. Cela signifie que nous cessons d'être à l'écoute. Lorsque nous sommes à l'écoute, cela nous permet de transformer un comportement négatif en comportement positif. Être à l'écoute est un élément important du fondement par lequel on peut regagner et recréer l'autodétermination.

Enfin, les enseignements spirituels de l'honnêteté et de la gentillesse pénètrent les cinq couleurs. L'honnêteté et la gentillesse sont des éléments qui prévalent dans ce système de croyances qui constitue le fondement sur lequel est fondé le concept d'autodétermination. C'est la première étape dans laquelle les ânés traditionnels jouent un rôle vital dans le processus qui mène à la compréhension de l'autodétermination au niveau communautaire ainsi qu'au niveau national.

\section{Conclusion}

Comment le fondement établi par les couleurs est-il lié à l'autodétermination? Au cours des dernières années, les aînés et les chefs ont commencé à promouvoir la guérison des communautés, en se servant de cérémonies traditionnelles, comme un des moyens disponibles pour que les communautés 
"La guérison, selon les traditions, crée des individus, des familles et des communautés plus puissantes, et cela favorise la résolution d'un grand nombre de problèmes sociaux et l'émergence, hors du contrôle $d u$ gouvernement fédéral, de nouvelles formes de développement social, économique et politique». commencent à prendre leurs responsabilités dans les domaines qu'elles considèrent importants. La guérison, selon les traditions, crée des individus, des familles et des communautés plus puissantes, et cela favorise la résolution d'un grand nombre de problèmes sociaux et l'émergence, hors du contrôle du gouvernement fédéral, de nouvelles formes de développement social, économique et politique. À ce jour, l'autonomie gouvernementale signifie que chacune des communautés contrôle et détermine le niveau d'autonomie gouvernementale qui lui est nécessaire. Mais ce n'est pas là une définition restrictive de ce qu'est ou de ce que pourra être l'autonomie gouvernementale des Amérindiens. Pour ceux et celles qui réagissent en disant que «les Amérindiens ne sont jamais contents», nous répondons que la transition d'une situation coloniale à une situation nationale prendra du temps. La nationalité amérindienne ne pourra pas se réaliser autrement qu'en fonction de l'identité amérindienne et la conception de sa place dans le monde, soit comme un don du Créateur. C'est pourquoi l'autonomie gouvernementale passe par une rappropriation de l'Esprit. Cela, chaque travailleur social euro-américain doit le comprendre et le respecter.

\section{Bibliographie}

ANGUS, Murry (1991). And the Last Shall Be First : Native Policy in an Era of Cutback, Toronto, NC Press Limited.

BOUDREAU, François (2000). «Identité, politique et spiritualité au nord du lac Huron : entretiens avec des leaders ojibwas», Recherches Amérindiennes au Québec, vol. XXX, no 1 (à paraitre).

BOUDREAU, François et François Xavier RIBORDY (1999). «Opération Rainbow : saga judiciaire sur fond de pluralisme juridique», dans F.X. Ribordy (sous la dir. de), La nature et la loi : le pluralisme juridique dans la gestion de la nature, Sudbury, Presses de l'Université Laurentienne, 141-175.

BROWN, Jennifer S.H. et Laura PEERAS (1970). The Chippewa and their Neibors : A Study in Ethnohistory, Prospect Heights, Illinois, Waveland Press Inc.

CHIEFS OF ONTARIO (1991). Signing the Statement of Political Relationship, Indian Associations Coordinating Committee of Ontario.

CLIFTON, James A. (1977). The Prairie People : Continuity and Change in Potawatomi Indian Culture 1665-1965, Laurence, The Regent Press of Kansas.

CRPA (1996a). «Un passé, un avenir», Rapport de la Commission Royale d'Enquête sur les Peuples Autochtones, Ottawa, Ministère des Approvisionnements et des Services du Canada, Groupe Communication Canada. 
CRPA (1996b). «Une relation à définir», Rapport de la Commission Royale d’Enquête sur les Peuples Autochtones, Ottawa, Ministère des Approvisionnements et des Services du Canada, Groupe Communication Canada.

CRPA (1996c). "Vers un ressourcement», Rapport de la Commission Royale d'Enquête sur les Peuples Autochtones, Ottawa, Ministère des Approvisionnements et des Services du Canada, Groupe Communication Canada.

CRPA (1996d). «Perspectives et réalités», Rapport de la Commission Royale d'Enquête sur les Peuples Autochtones, Ottawa, Ministère des Approvisionnements et des Services du Canada, Groupe Communication Canada.

CRPA (1996e). "Vingt ans d'action soutenue pour le renouveau», Rapport de la Commission Royale d'Enquête sur les Peuples Autochtones, Ottawa, Ministère des Approvisionnements et des Services du Canada, Groupe Communication Canada.

CRPA (1995). "Choisir la vie», Un rapport spécial sur le suicide chez les autochtones, Ottawa, Ministère des Approvisionnements et des Services du Canada, Groupe Communication Canada.

DENEVAN, W.M. (1976). The Native Population of the America in 1492, Madison, University of Wisconsin Press.

GHOSTKEEPER, Elmer (1996). Spirit Gifting: The Concept of Spiritual Exchange, Calgary, The Artic Institute of North America, The University of Calgary.

HALLOWELL, Irving, A. (1967). Culture and Experience, New York, Schocken Books.

JILES, Paulette (1995). North Spirit :Travels Among The Cree and Ojibway Nations and Their Star Map, Toronto, Doubleday Canada Limited.

KICKINGBIRD, Kirke et al. (1977). Indian Sovereingty, Washington, DC., Institute for the Development of Indian Law.

KNOX, R.H.(1990). Indian Conditions :A Survey, Ottawa, Department of Indian Affairs and Northern Development.

NABIGON, Herb (1992). «Reclaiming the Spirit for First Nations Self Government», dans AnneMarie Mawhiney (sous la dir. de), Rebirth: Political, Economic and Social Development in First Nations, Toronto et Oxford, Dundurn Press, 136-145.

PLATIEL, Rudy (1992). «Canada Reconsidered : Aboriginal Rights», Globe and Mail, 11 January, A6.

ROSS, Heather (1987). First Nations Self-Government, Background Report, Prepared for the Social Assistance Review Committee, Toronto, Ontario.

SCHMALZ, Peter S. (1991). The Ojibwa of Southern Ontario, Toronto, University of Toronto Press.

SHKILNYK,Anastasia M. (1985). A Poison StrongerThan Love :The Destruction of an Ojibwa Community, New Haven and London, Yale University Press.

SPIELMAN, Roger (1998). «You're So Fat» : Exploring Ojibwe Discourse, Toronto, University of Toronto Press.

UBELAKER, D. (1976). «The Sources and Methodology for Mooney's Estimates of North American Indians Populations», dans W.M. Denevan (sous la dir. de), The Natives Population of the Americas in 1492, Madison, University of Wisconsin Press.

VAN DE SANDE, A. et G. RENAULT (1998). «L'intégration des concepts autochtones dans le curriculum du travail social», Reflets, vol. 4, no 1,164-173.

WARREN, William (1974) [1885]. History of the Ojibway Nation, Minneapolis, Minnesota, Ross \& Haines Inc. 


\section{Notes}

1. Ce texte présente une conception traditionaliste de l'autonomie gouvernementale. Par traditionaliste, nous entendons une position politique informée par les aînés et par la tradition, surtout dans ses mythes et dans sa spiritualité.

2. Le concept Anishnabee ou Anishinaabe signifie les gens d'ici ou encore nous les Hommes de cette terre, que Warren traduit par l'homme spontané, (1974:56), dénomination reprise par Jiles (1995 : 2). Notons également les orthographes Anishnabai utilisé par les Ojibwas de Temagami, Nishnaabe utilisé par la section amérindienne de l'École de service social de l'Université Laurentienne, Anishnaabe (Spielmann 1998), Neshnabē (Clifton 1977), Änicinábek (Hallowell 1967) et Anišsina'pe' (Browns et Peers 1970), toutes ses formes se traduisent, en un sens générique, par «êtres humains». Ce concept est de plus en plus utilisé par les Ojibwas pour s'identifier euxmêmes. C'est donc le concept que nous utilisons pour parler des Ojibwas.

3. Nous entendons par populations euro-américaines, les populations et les cultures venues d'Europe, de France et d'Angleterre surtout, qui se sont implantées en Amérique avec leurs valeurs et leurs institutions.

4. Il faut souligner le problème que cet établissement territorial a posé à l'État. En effet, il correspondait à la volonté étatique de sédentariser les Amérindiens. Par contre, cette sédentarisation s'accompagnait d'une forme de reconnaissance territoriale et politique des Métis et des Amérindiens (Cris). D'une certaine manière, c'est ce qui deviendra de plus en plus inacceptable pour l'État central, c'est de voir la constitution de cette entité politique à la frontière du Haut Canada, là où précisément l'État voulait étendre sa mainmise pour favoriser l'établissement de colons «blancs». L'État voulait certes que les Amérindiens se sédentarisent, mais en petits groupes isolés ça et là. Il ne voulait surtout pas que les Amérindiens s'établissent globalement comme force politique autonome sur un vaste territoire.

5. Cette décision est une reconnaissance de principe, sans support législatif, de la part du gouvernement néo-démocrate de l'Ontario qui :a) reconnaît les Premières Nations en tant que nations distinctes avec le droit à l'autodétermination, b) établit une relation de gouvernement à gouvernement entre les Premières Nations et le gouvernement de l'Ontario et, c) qui engage le gouvernement de l'Ontario à négocier l'exercice de juridictions à concéder (Chiefs of Ontario 1991).

6. En mai 1990, la Cour Suprême du Canada reconnaît et affirme que les droits aborigènes et les traités signés font partie intégrante de la Constitution du Canada (1982, sect. 35, [1]). Dans cette décision, la Cour considère que la conservation des ressources va de pair avec leur exploitation et qu'à ce titre, les membres des Premières Nations, premiers exploitants des ressources, ont priorité sur les ressources. En cas de conflit, la Cour Suprême encourage la négociation avec les peuples autochtones. La couronne a la responsabilité fiduciaire, elle doit agir dans la confiance plutôt que dans l'adversité. Cette décision n'est cependant pas toujours respectée (Boudreau et Ribordy, 1999).

7. Les déplacements forcés des populations amérindiennes depuis le premier contact sont nombreux : des Hurons par les Français, au XVII ${ }^{\mathrm{e}}$ siècle, suite aux guerres tribales contre les Cinq Nations, le déplacement des Cinq Nations et des Potawatomies après la guerre canado-américaine de 
1814 ou, plus récemment, le déplacement d'Innus du nord du Québec aux îles de Baffin par le gouvernement fédéral qui voulait ainsi assurer une présence autochtone pour affirmer la souveraineté du Canada sur les îles de la mer Arctique ou le déplacement des Cris suite à l'entente de la baie James en 1974.

8. Sur le paradigme culturel, sur l'usage de la roue médicinale en service social ou une autre version des quatre directions, voir van de Sande et Renault (1998:164-173). 